\title{
Radiological Evaluation of Postsurgical Course of Perforators in Retrograde Posterior Tibial Fasciocutaneous Flaps for Distal Leg and Foot Defects
}

\author{
Neeraj Kant Agrawal ${ }^{1}$ Visweswar Bhattacharya ${ }^{1} \quad$ Rahul Dubepuria ${ }^{2}$ \\ 1Department of Plastic Surgery, Institute of Medical Sciences, \\ Banaras Hindu University, Varanasi, Uttar Pradesh, India \\ 2Department of General Surgery, Sri Aurobindo Institute of Medical \\ Sciences, Indore, Madhya Pradesh, India \\ Address for correspondence Neeraj Kant Agrawal, MCh, MS, 704, \\ Rudra Samriddhi, Bhagwanpur, Varanasi, Uttar Pradesh, 221005, \\ India (e-mail: drneerajplasticsurgery@gmail.com). \\ Indian J Plast Surg 2021;54:272-277.
}

\author{
Abstract \\ Keywords \\ - posterior tibial \\ perforators \\ - fasciocutaneous flaps \\ - pedicled flaps \\ - perforator assessment \\ - CTA \\ - vascular mapping
}

Background Mapping of vascular perforators by various methodologies have been described for planning of a variety of flaps in the lower limbs. We attempted to assess the changes in posterior tibial perforators after transfer of fasciocutaneous flaps for leg defects.

Methods 20 patients with distal leg and foot defects were studied by computed tomography angiography (CTA) and preoperative audio Doppler to ascertain perforators of posterior tibial artery. Fasciocutaneous flaps were raised, based on these perforators, depending on the site and size of soft-tissue defects. The number of perforators and their distance from the medial malleolus were also studied. Postoperative CTA was performed on the 7th to 10th day, with emphasis on postoperative changes of the perforators on which the flaps were based.

Results One to four posterior tibial perforators were found between $5 \mathrm{~cm}$ and $8 \mathrm{~cm}$ proximal to the medial malleolus. After flap transfer, the perforators could be traced to variable distance through the total length of the flap. The perforators formed small vascular loop in 12 patients, following retrograde posterior tibial flap transfer. The height of the loop, the number of such loops, the dilatation and tortuosity of the perforators, and their longitudinal orientation were studied in detail. Most of the findings can be explained by mechanical realignment of perforators as well as by the delay phenomenon associated with retrograde fasciocutaneous flaps.

Conclusion It was concluded that the morphological changes associated with the perforators explained the vascular rationality and success of these flaps.

\section{Introduction}

Deep fascia of lower limbs has been used as vascular carrier in fasciocutaneous flaps for about four decades. Posterior tibial artery and its perforators play a vital role in forming the vascular arcade in deep fascia and subsequently communicating

published online September 2, 2021
DOI https://doi.org/

$10.1055 / \mathrm{s}-0041-1734568$ ISSN 0970-0358 with subcutaneous and subdermal plexus. ${ }^{1}$ Vascularity is principally derived from septocutaneous perforators that emerge from the posterior tibial artery at 4 to $26 \mathrm{~cm}$ from the medial malleolus. ${ }^{2}$ Musculocutaneous and septomusculocutaneous perforators have also been described. However, only septocutaneous perforators are present in the distal third (c) 2021. Association of Plastic Surgeons of India.

This is an open access article published by Thieme under the terms of the Creative Commons Attribution-NonDerivative-NonCommercial-License, permitting copying and reproduction so long as the original work is given appropriate credit. Contents may not be used for commercial purposes, or adapted, remixed, transformed or built upon. (https://creativecommons.org/licenses/by-nc-nd/4.0/).

Thieme Medical and Scientific Publishers Pvt. Ltd. A-12, 2nd Floor, Sector 2, Noida-201301 UP, India 
of the leg. ${ }^{3}$ Each arterial perforator is accompanied by two venae comitantes, draining into the great saphenous vein. The rich subdermal axial network allows dissection of large meticulously designed flaps based on these perforators. ${ }^{4}$

Objective mapping of the perforators has been defined in cadavers as well as living beings. In cadavers, mapping of perforators has been described, using radiopaque injections like barium sulfate and lead oxide. ${ }^{5,6}$ Computed tomography angiography (CTA) completed the gamut of perforator imaging, which was earlier assessed only by color Doppler ultrasonography (CDU). A number of researchers have used CTA for preoperative perforator imaging. ${ }^{7}$ However, the vascular anatomy of the perforators following the transfer of flaps has been inadequately studied. Postsurgical alterations in the perforator anatomy are specific and need detailed evaluation by CTA.

We aimed to study the postoperative course of the perforators on which the flap was planned and raised. We analyzed the subject to provide a logical explanation to the exclusive findings.

\section{Material and Methods}

From October 2018 to March 2020, 20 patients with skin and soft-tissue defects of the distal third of leg, ankle, and foot were admitted for treatment in the department of plastic surgery at an apex trauma center. They were resurfaced by retrograde posterior tibial perforator-based flaps. The study was undertaken after obtaining approval from the Institute Ethics Committee and following informed consent from the subjects.

Injury to major vessels of the lower extremity was ruled out. Supple and pain-free movements of the ankle joints were important for early mobilization. Patients with profusely discharging wounds were included only after the discharge decreases or ceases. Subjects with significant medical illness as well as those who were addicted to smoking were also excluded from the study.

Meticulous preoperative assessment of wounds was done prior to surgery. Wound was assessed carefully for edema of edges, obvious necrotic tissue, and structures exposed in the floor. Defect dimensions were measured and availability of relevant donor tissue was evaluated. Dimensions of the tentative posterior tibial fasciocutaneous flap and the tentative incision site were also noted. Subjects with extensive locoregional scarring encroaching the donor area of posterior tibial flaps were excluded. Strict blood glucose control and evaluation of coagulation function were vital to prevent complications following surgery. Incisional tissue biopsy helped in culture-based antibiotic treatment in chronically infected patients.

Color Doppler (EPIQ Elite, Royal Philips Electronic Inc, Amsterdam, Netherlands), was performed with $7.5 \mathrm{MHz}$ to $11.5 \mathrm{MHz}$ linear probes. It helped in locating the site, length, direction, and internal diameter of the perforators. The investigative modality of choice was a 128 slice CT Angiographic evaluation, which was performed using a nonionic iodine-based dye (Discovery MI DR, GE Health care, Milwaukee, USA). Images were obtained and processed for visualization of peripheral vasculature, including the perforators that would be incorporated in the base of the flap.
They were reconstructed at various angles and rotation. The images were processed using volume-rendering and maximum projection intensity functions to select and hide desired regions of the image which enabled detailed high-contrast evaluation of the region in three dimensions.

Vascular perforators identified by radiological evaluation were confirmed by handheld audio Doppler just prior to the surgery. Perforators were marked along the vascular axis which was marked approximately $2.5 \mathrm{~cm}$ posterior to the medial border of tibia. Careful planning was of utmost importance to raise an adequate flap. The wound was prepared by thorough debridement and rigorous cleaning.

Retrograde fasciocutaneous flaps were dissected, depending upon the site and size of the soft-tissue defect. The anatomical distribution of soft-tissue defects is depicted in - Table $\mathbf{1}$. The patients were operated in supine position with hip externally rotated for easy harvesting of flap from the medial side of leg. The proximal limit of the flap was marked $10 \mathrm{~cm}$ distal to the knee joint. Under tourniquet control, dissection of the planned flap was initiated by proximal and anterior incision. As the flap was raised from proximal to distal, perforators were encountered, tethered to the deep fascia, which were severed, except for the distal one or two perforators, and would be included in the flap pedicle. The pedicle was also narrowed toward the base of the flap, keeping 4 to $5 \mathrm{~cm}$ width and ascertaining the inclusion of perforators ( $\boldsymbol{- \text { Fig. }} \mathbf{1}$ ). In addition, it was beneficial to free the perforator from the surrounding tissues to diminish the prospect of vascular kinking and subsequent complications. This also permitted smooth transfer of the flap with a relaxed pedicle. After elevation and retrograde transfer of the flap, tension-free suturing of the flap was done to prevent tissue ischemia. A noncompressive windowed dressing was applied for daily monitoring of the flap. The limb was elevated above the heart level to minimize postoperative edema. The perforators that were marked preoperatively would be further evaluated in the postoperative period utilizing CTA.

It was performed after 7 to 10 days of flap transfer. The posterior tibial vessel and the perforators, on which the flaps were based, were evaluated and vascular changes looked for. The perforator course through the length of the flap was analyzed in the postoperative period.

\section{Results}

Average age of the 20 patients was $36.85 \pm 12.71$ years (range 18-60 years). Half of the patients were aged 35 years or less while $90 \%$ were males (-Table $\mathbf{1}$ ). The average size of posterior tibial perforator-based flaps to resurface wounds was $105.27 \mathrm{~cm}^{2}$ flaps $\left(45-200 \mathrm{~cm}^{2}\right)$, and they were operated in 132.37 minutes. All the 20 patients were evaluated for vascularity and imaging of posterior tibial perforators in the postoperative period.

As much as $50 \%$ of the patients sustained wounds due to trauma followed by nonhealing ulcer including diabetic ulcer (35\%). Tissue defects on the distal third of leg was found in 10 patients (50\%), over the dorsum of foot in four patients, over medial malleolus in three, and on weight-bearing heel in two patients ( - Table $\mathbf{1}$ ). Half of the patients had unhealthy 

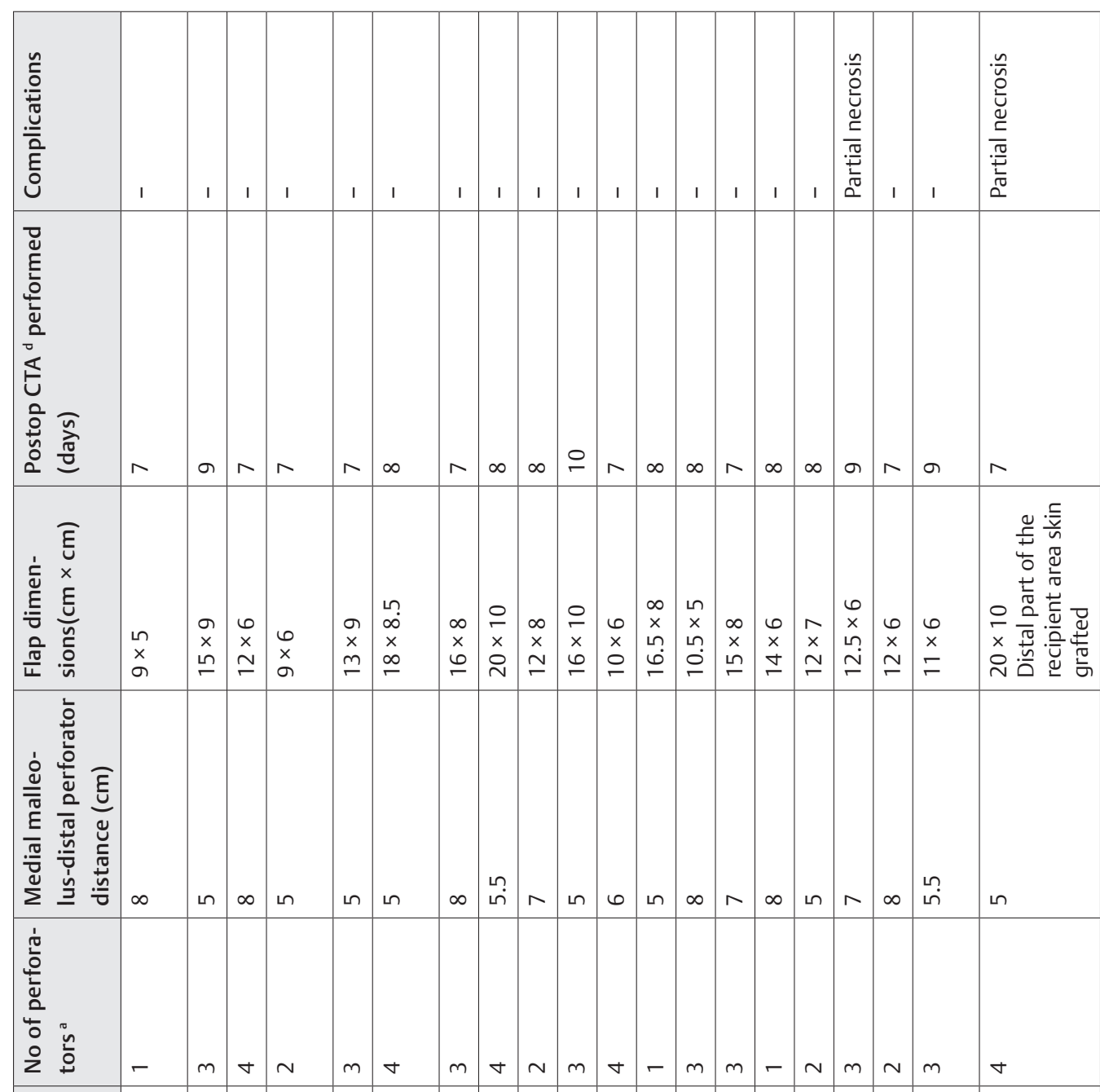

\begin{tabular}{|c|c|c|c|c|c|c|c|c|c|c|c|c|c|c|c|c|c|c|}
\hline 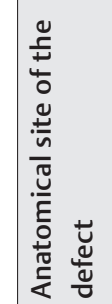 & 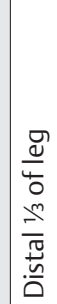 & 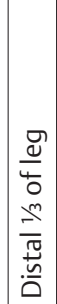 & 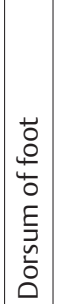 & 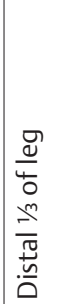 & 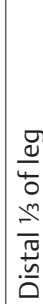 & 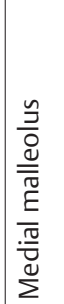 & $\begin{array}{l}\frac{g}{4} \\
\frac{\pi}{0} \\
\frac{m}{\pi} \\
\frac{\pi}{0}\end{array}$ & 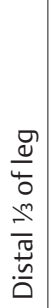 & 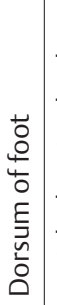 & $=$ & 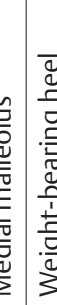 & 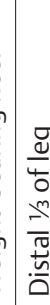 & 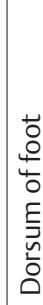 & 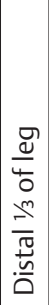 & & 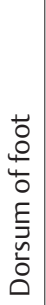 & & 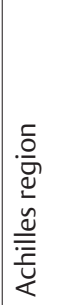 \\
\hline
\end{tabular}

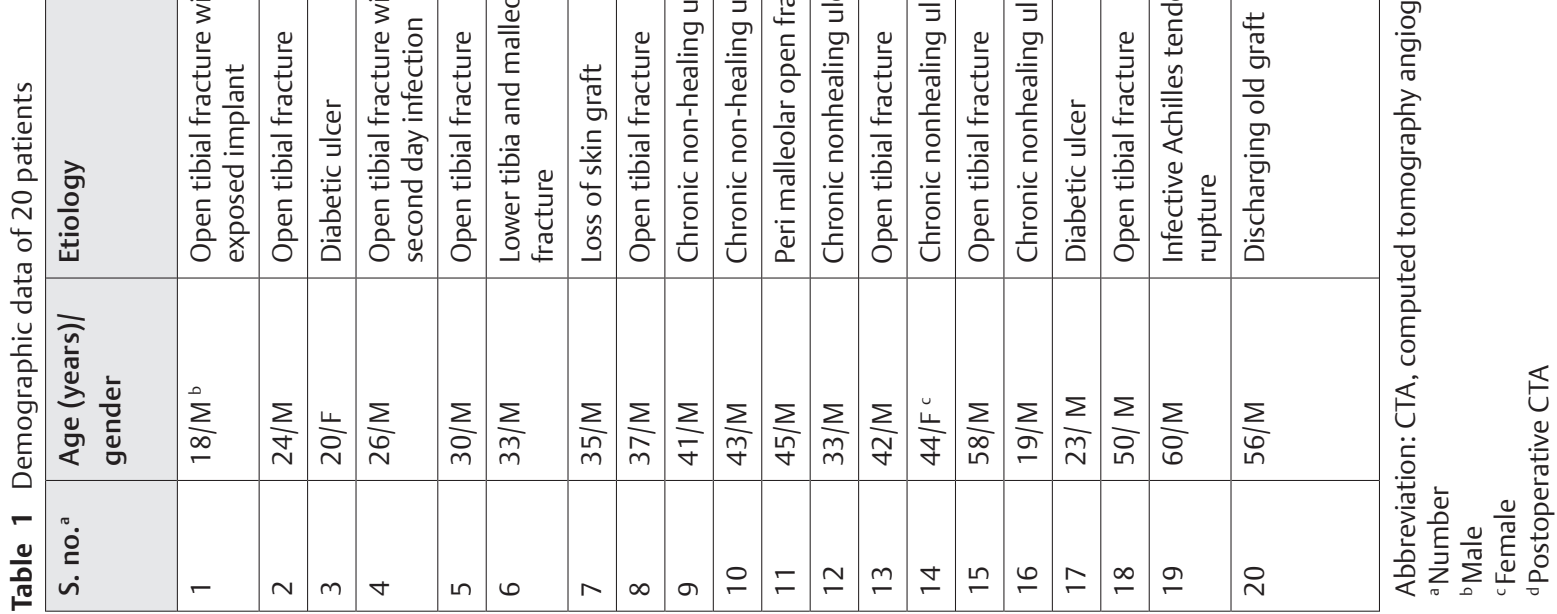


wounds and required prolonged antibiotic dressing and sequential debridement before definitive resurfacing.

Preoperative CTA of the involved lower limb was performed to assess vascular axis of the posterior tibial artery and its perforators. The variables studied on preoperative CTA were the number of perforators, which were $2.75 \pm 1$ (range 1-4, median 3) ( - Fig. 2), and medial malleolus to the distal most perforator distance, which was $6.3 \pm 1.3 \mathrm{~cm}$ (range 5-8 cm, median 5.75) ( - Fig. 3). The perforators were single in three cases and multiple in 17 patients, and 50\% of the posterior tibial perforators were clustered within $5-6 \mathrm{~cm}$

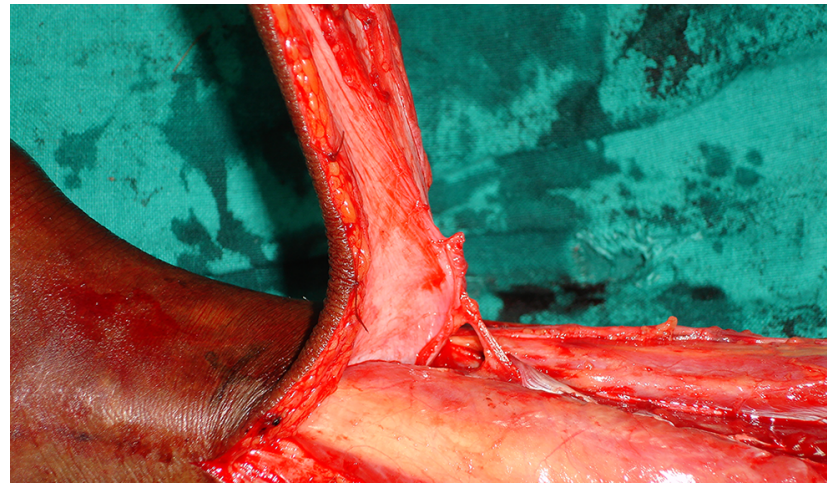

Fig. 1 Perforator emerging from the posterior tibial artery, accompanied by venae comitantes in a pedicled fasciocutaneous flap.

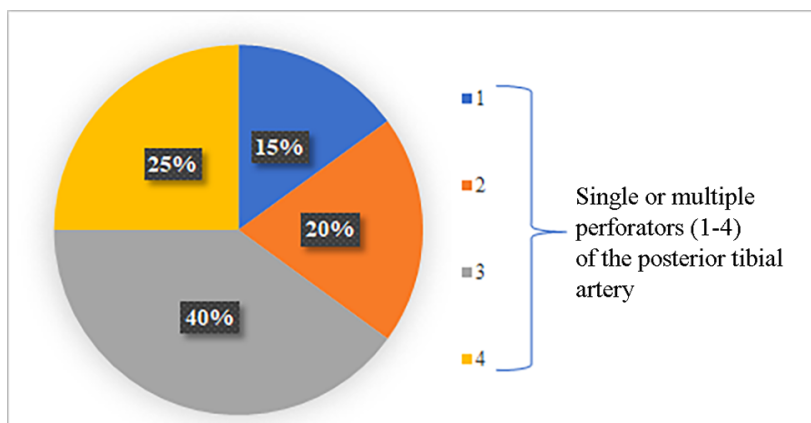

Distribution of perforators in the 20 patients

Fig. 2 Pie chart depicting the number of perforators from the posterior tibial artery, with $40 \%$ lower limbs having three perforators.

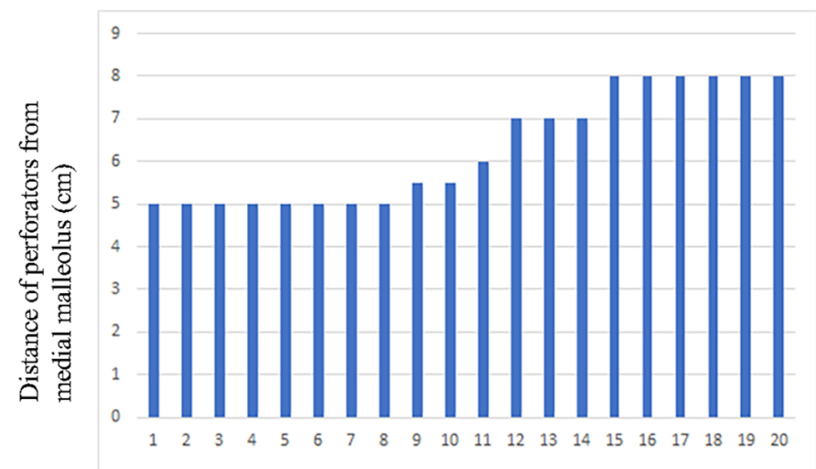

Distribution in 20 patients of posterior tibial perforator based flaps

Fig. 3 Bar diagram depicting the site of posterior tibial perforators, especially found at $5-6 \mathrm{~cm}$ proximal to the tip of medial malleolus.
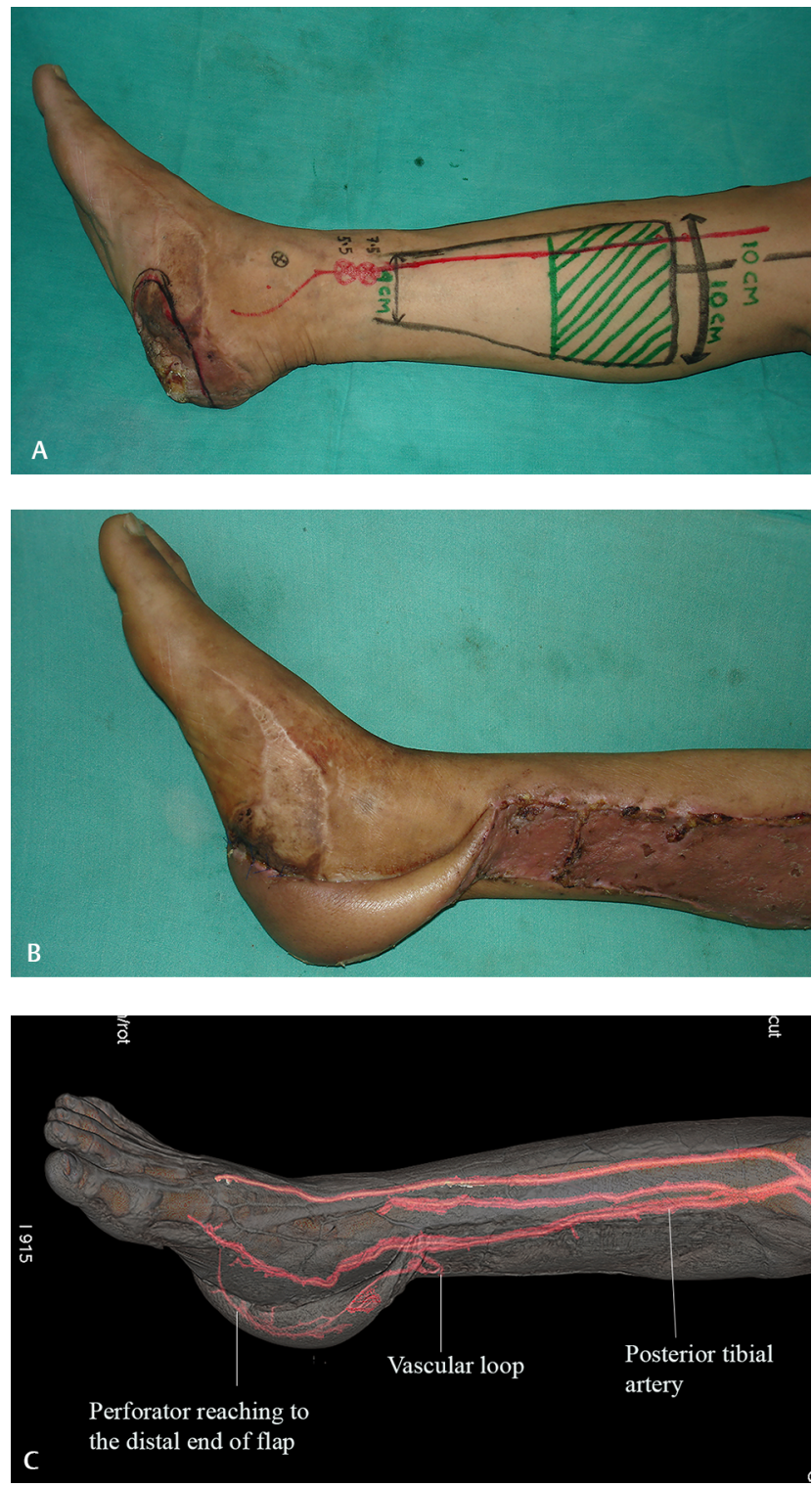

Fig. 4 (a) Reconstruction of right heel by $16 \times 10 \mathrm{~cm}$ retrograde pedicled fasciocutaneous flap, based on perforators of the posterior tibial artery at $5.5 \mathrm{~cm}$ and $7.5 \mathrm{~cm}$ from the medial malleolus (serial no. 10 in Table 1). The base of the flap was narrowed to $4 \mathrm{~cm}$. (b) Flap raised, preserving the perforators, transferred and inset. (c) Computed tomography angiography (CTA) showed distal perforator forming a small vascular loop and coursed tortuously to the distal most sutured portion of the flap.

from the medial malleolus ( $\mathbf{- T a b l e} \mathbf{1}$ ). Flaps survived well in 18 patients (90\%), while two patients had partial necrosis.

Postoperative CTA was performed at a median of 8 days ( 7 to 10 days) after surgery to study the perforators following flap transfer. The perforators were traced through the flap up to the distal most part. Dilatation of perforators as well as tortuosity were observed following flap transfer. In six cases, we found that the perforators reached almost to the distal end of the flap, while in 12 patients, they traversed 50 to $60 \%$ of the flap length. However, in two cases, they could be traced up to less than $50 \%$ of the total flap length. In addition, perforators after emerging from the main trunk formed small vascular loops in 12 out of 20 patients (60\%) (-Figs 4-6). 

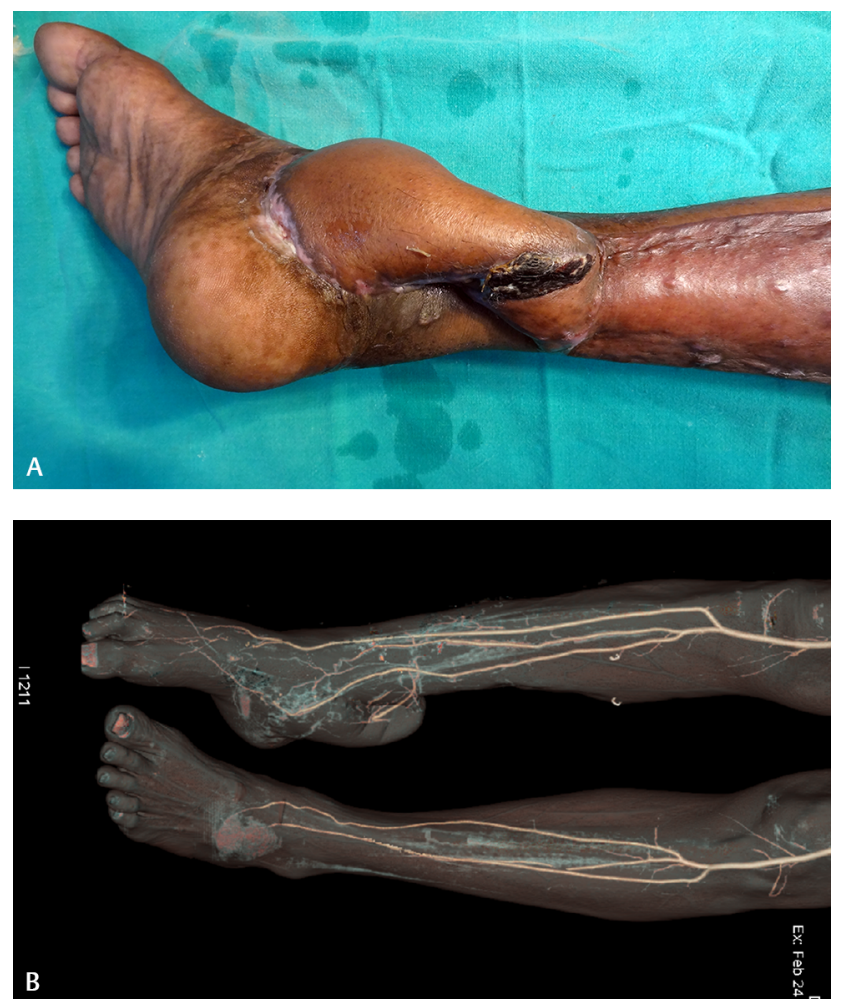

Fig. 5 (a) Soft-tissue defect over right medial malleolus resurfaced by $12 \times 7 \mathrm{~cm}$ retrograde posterior tibial perforator-based fasciocutaneous flap (serial no. 16 in Table 1). (b) Computed tomography angiography (CTA) depicted the perforator course through the length of the flap.

\section{Discussion}

Precise understanding of the vascularity of fasciocutaneous flaps has fascinated scientific workers across the globe. Dynamic infrared thermography has surfaced as a noninvasive means of postoperative flap assessment, but it does not provide any information about perforators. ${ }^{8}$ Traditional angiography was considered the gold standard of imaging for many years. CDU can provide substantial information, but it is real-time, operator-dependent, and unable to produce a three-dimensional image of the vasculature. ${ }^{9} \mathrm{CTA}$ imaging of the posterior tibial perforators was performed to provide significant understanding about the mechanism of survival of fasciocutaneous flaps.

CTA has been frequently used for planning of perforator flaps but has not been applied to study the perforator course in the transferred pedicled flaps. It was observed that the perforators, after they emerge from the posterior tibial vessels, formed small vascular loops following flap transfer. This can well be explained by mechanical realignment of the flap as the tissue moved almost $180^{\circ}$ at the base of the flap. Identification of perforators and their mobilization prevented unnecessary torsion. Retrograde flaps demonstrated distinct perforator course in the superficial plane of the flap. This is in accordance with the "Gent" consensus that explained perforator terminologies. It also described that perforators traverse deep fascia and supply the dermal plexus, thus, forming the vascular basis of these flaps. ${ }^{10}$
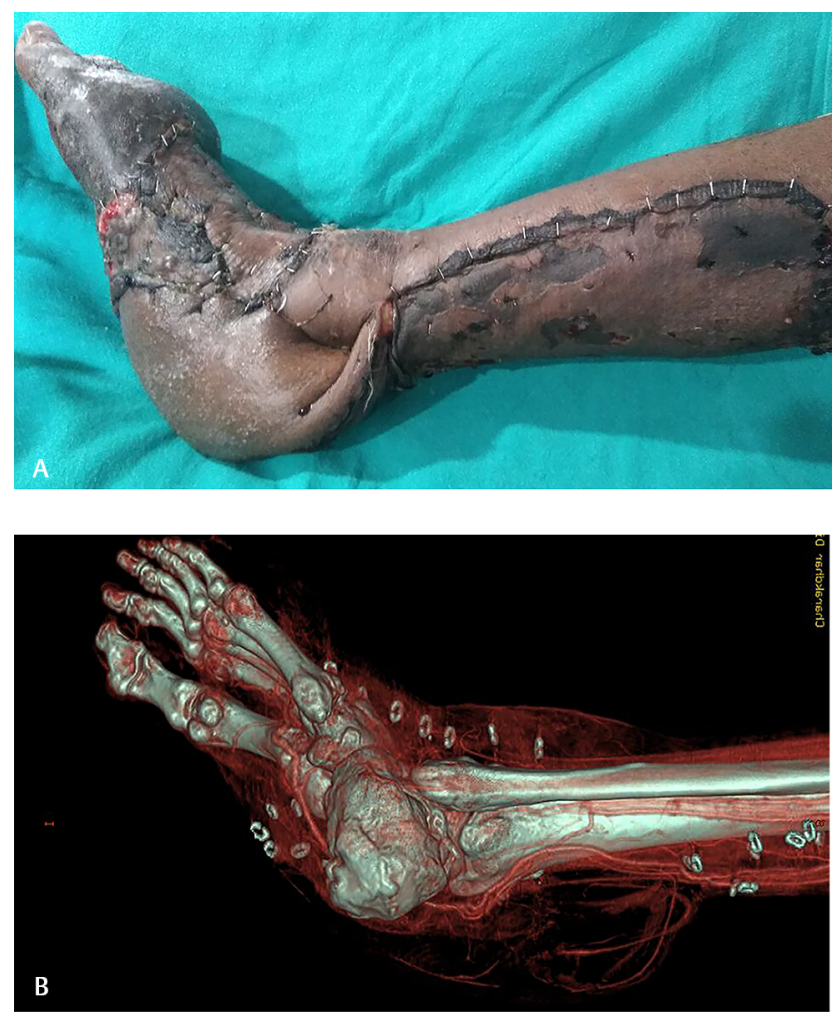

Fig. 6 (a) Profusely discharging skin graft over the dorsum of right foot and part of heel, necessitating excision and flap cover, using $16.5 \times 8 \mathrm{~cm}$ posterior tibial perforator-based flap (serial no. 12 in Table 1). Two perforators, at $5 \mathrm{~cm}$ and $8 \mathrm{~cm}$ from the medial malleolus, were preserved. (b) Computed tomography angiography (CTA) beautifully illustrated both the retained perforators and their postsurgical course through the flap. They could be seen coursing to variable distances distally along the entire flap length.

The height of the vascular loop depended on the laxity of the flap at its base. The number of loops may be one or more, which was determined by the number of perforators retained in the base of the flap. Posterior tibial artery contributed sizeable perforators with perforator course along the length of the flap, independent of caliber of the perforators. Retrograde fasciocutaneous flaps, which are, in effect, delayed flaps, demonstrated dilatation of existing vessels within the flap and not ingrowth of new vessels. ${ }^{11}$ Such surgical delay was responsible for longitudinal orientation of perforators, as evidenced by postoperative CTA.

The relative ischemia of the flap, induced by the surgical delay, was further confirmed by the vivid perforator course through the flap. The perforators, after originating from the vascular trunk, were traced up to different distances along the long axis of the flap ( $\boldsymbol{- F i g}$. 4c). Ischemia-driven vascular dilatation assisted in better visualization of the perforators and their subdermal course. The perforators that reach almost to the distal end of the flap indicate a well-dilated and adequately perfused flap. The entire subdermal course was seen, and this concept has been highlighted by researchers. ${ }^{12}$ The timing of postoperative CTA was not decided arbitrarily. Tsur et al in their early work demonstrated neovascularization from the recipient bed and margins within 7 to 10 days of intervention. ${ }^{13}$ The CTA would give best results before 
neovascular contribution and, therefore, was performed at a median of the 8th postoperative day.

Studies have also shown that the flap elevation resulted in increase of the diameter of choke vessels, which link adjacent vascular territories or angiosomes, on the 3rd day to reach a maximum at day 5 . Their tortuosity increased as well, leading to increased vascular length, the change occurring from day 5 until day $7 .^{14}$

Posterior tibial fasciocutaneous flap was earlier supposed to depend on the vascular supply of the direct cutaneous branches of the posterior tibial vessels. Carriquiry et al reported that there are three main groups of septocutaneous perforators, located at 9 to 12,17 to 19 , and 23 to $32 \mathrm{~cm}$ from the tip of the medial malleolus. ${ }^{15}$ On the contrary, Blondeel categorized posterior tibial perforators as primarily musculocutaneous. ${ }^{10}$ Schaverien in his work deduced that perforators arising from the posterior tibial arteries were found within discrete septa. Reliable perforators of the posterior tibial artery were found within three distinct $5-\mathrm{cm}$ intervals at 4 to $9 \mathrm{~cm}, 13$ to $18 \mathrm{~cm}$, and 21 to $26 \mathrm{~cm}$ from the medial malleolus. ${ }^{2}$ We demonstrated sizable, distal-most perforators of vessels on which flaps were raised. In our study, the posterior tibial perforators were found between $5 \mathrm{~cm}$ and $8 \mathrm{~cm}$ (median of $7 \mathrm{~cm}$ ) from the medial malleolus. Thus, the findings corroborate well with those of Schaverien. This knowledge of the location of perforators is of paramount importance while planning a posterior tibial flap and enhancing the success rate of the fasciocutaneous flaps. Bulla et al demonstrated that the mean distance of the perforator from the medial tibial border was $1.23 \mathrm{~cm}$ as opposed to the conventional $2.5 \mathrm{~cm} .{ }^{16}$ In the present work, the number of posterior tibial perforators was a median of $3(1-4)$. However, cadaveric dissection of 155 perforators of posterior tibial artery demonstrated an average number of five perforators. ${ }^{3}$

Haertsch reported strikingly deficient perforating vessels in the lower leg. ${ }^{17}$ However, we could find a sufficient number of perforators of the posterior tibial vessels with appropriate diameter in the middle and distal third of leg. Thinner modifications of fasciocutaneous flaps such as adipofascial or fascial flaps have limited usefulness, owing to smaller dimensions. ${ }^{18,19}$ Posterior tibial perforator-based flaps can be used for reconstruction of massive defects, and it is better than other more complex microvascular techniques of flap transfer. ${ }^{20}$ The versatile retrograde posterior tibial perforator-based fasciocutaneous flaps is a simple technique that remains the backbone of reconstruction of distal leg and foot defects.

\section{Conclusion}

Retrograde fasciocutaneous flaps, based on radiologically confirmed perforators of posterior tibial artery, were successfully executed for distal leg and foot defects. CTA was helpful to analyze the course of perforators, following flap transfer, as well as ascertain the viability of the flap. The morphological changes in the vasculature were unique and add to the existing knowledge.

Conflict of Interest

None declared.

\section{References}

1 Cormack GC, Lamberty BG. A classification of fascio-cutaneous flaps according to their patterns of vascularisation. Br J Plast Surg 1984;37(1):80-87

2 Schaverien M, Saint-Cyr M. Perforators of the lower leg: analysis of perforator locations and clinical application for pedicled perforator flaps. Plast Reconstr Surg 2008;122(1):161-170

3 Drimouras G, Kostopoulos E, Agiannidis C, et al. Redefining vascular anatomy of posterior tibial artery perforators: a cadaveric study and review of the literature. Ann Plast Surg 2016;76(6):705-712

4 Heymans O, Verhelle N, Peters S. The medial adiposofascial flap of the leg: anatomical basis and clinical applications. Plast Reconstr Surg 2005;115(3):793-801

5 Tan BK, Ng RT, Tay NS, Tan BS. Tissue microangiography using a simplified barium sulphate cadaver injection technique. Ann Acad Med Singapore 1999;28(1):152-154

6 Rees MJ, Taylor GI. A simplified lead oxide cadaver injection technique. Plast Reconstr Surg 1986;77(1):141-145

7 Ribuffo D, Atzeni M, Saba L, et al. Clinical study of peroneal artery perforators with computed tomographic angiography: implications for fibular flap harvest. Surg Radiol Anat 2010;32(4):329-334

8 Shejbal D, Drvis P, Bedekovic V. Thermography-measured effect of capsaicin, methylprednisolone and mitomycin on the survival of random skin flaps in rats. Skin Res Technol 2012;18(2):157-161

9 Gravvanis A, Petrocheilou G, Tsoutsos D, Delikonstantinou I, Karakitsos D. Integrating imaging techniques in lower limb microsurgical reconstruction: focusing on ultrasonography versus computed tomography angiography. In Vivo 2013;27 (3):371-375

10 Blondeel PN, Van Landuyt KH, Monstrey SJ, et al. The "Gent" consensus on perforator flap terminology: preliminary definitions. Plast Reconstr Surg 2003;112(5):1378-1383, quiz 1383, 1516, discussion 1384-1387

11 Dhar SC, Taylor GI. The delay phenomenon: the story unfolds. Plast Reconstr Surg 1999;104(7):2079-2091

12 Bhattacharya V, Agrawal NK, Chaudhary GR, Arvind S, Bhattacharya S. CT angiographic evaluation of perforators in the lower limb and their reconstructive implication. Indian J Plast Surg 2012;45(3):494-497

13 Tsur H, Daniller A, Strauch B. Neovascularization of skin flaps: route and timing. Plast Reconstr Surg 1980;66(1):85-90

14 Zhuang Y, Hu S, Wu D, Tang M, Xu DC. A novel in vivo technique for observations of choke vessels in a rat skin flap model. Plast Reconstr Surg 2012;130(2):308-317

15 Carriquiry C, Aparecida Costa M, Vasconez LO. An anatomic study of the septocutaneous vessels of the leg. Plast Reconstr Surg 1985;76(3):354-363

16 Bulla A, De Luca L, Campus GV, Rubino C, Montella A, Casoli V. The localization of the distal perforators of posterior tibial artery: a cadaveric study for the correct planning of medial adipofascial flaps. Surg Radiol Anat 2015;37(1):19-25

17 Haertsch PA. The blood supply to the skin of the leg: a post-mortem investigation. Br J Plast Surg 1981;34(4):470-477

18 Bhattacharya V, Goyal S, Bashir SA. Reconstructive implications of adipofascial flaps in limb defects. Eur J Plast Surg 2007;30:169-175

19 Agrawal NK, Gupta M. Distally based non-axial fascial hinged flaps: useful adjunct to resurface traumatic soft tissue defects of the distal leg. Int J Contemp Med Res 2020;7(6):1-6

20 Yang X, Chen G, Zeng H, Wang W, Chen Y, Li Z. Distal posterior tibial artery perforator flaps for the treatment of chronic lower extremity wounds. Int JClin Exp Med 2016;9(10):19553-19560 